\title{
Hormonioterapia oral no câncer de mama: fatores que podem influenciar na adesão
}

Oral hormone therapy in breast cancer: factors that can influence adherence Terapía hormonal oral en el câncer de mama: factores que pueden influenciar la adhesión

Martamaria de Souza Ferraz Ribeiro ${ }^{1}$; Marcel Tavares de Farias $^{2}$; Islania Almeida Brandão ${ }^{3}$; Patrícia Alcântara Doval de Carvalho Viana ${ }^{4}$

\section{Resumo}

A adesão terapêutica é definida como o grau de cumprimento das instruções médicas pelo paciente. A terapia endócrina ou hormonioterapia consiste no uso de substâncias semelhantes a hormônios, ou supressoras desses, para inibir o crescimento tumoral. É um tratamento de longa duração, com efeitos adversos importantes, o que implica em dificuldade de adesão. Neste estudo foram utilizados dois métodos para avaliar a adesão, o teste Morisky e Green e o questionário proposto por Marques. De acordo aos resultados do teste Morisky e Green, $80 \%$ dos pacientes entrevistados foram considerados não aderentes, enquanto, por meio do questionário de Marques, analisando os fatores que podem influenciar a não adesão, foi detectada uma média de pontos de $24,83 \pm 4,84$, que corresponde ao resultado de nenhuma dificuldade em aderir ao tratamento. Houve diferença nos resultados, porém as metodologias de avaliação e a elucidação das informações extraídas diferem de tal modo, que cada método deve ser analisado separadamente e criticado dentro de suas limitações. Foi possível inferir que o teste Morisky e Green é útil para nortear uma avaliação, porém não reflete bem o grau de adesão, uma vez que tende a superestimar a não adesão. Todavia, avaliar os fatores que podem influenciar a adesão, como proposto por Marques, é uma maneira útil de detectar as dificuldades dos pacientes em aderir ao tratamento medicamentoso, a fim de influenciar de maneira positiva a adesão.

Descritores: Neoplasias de mama, Adesão à Medicação, Avaliação, Institutos de Câncer, Neoplasias Hormônio-Dependentes.

\section{Abstract}

Therapy adherence is defined as the degree of compliance with medical instructions by the patient. Endocrine therapy or hormonal therapy is the use of hormone-like substances or these suppressors, to inhibit tumor growth. It is a long-term treatment, with significant adverse effects, which implies in difficulty of adherence. In this study two methods were used to evaluate adherence, the Morisky test and Green and the questionnaire proposed by Marques. According to Morisky and Green test results, $80 \%$ of interviewed patients were considered non-adherent while through Marques

\footnotetext{
1 Especialista em Farmácia em Oncologia pela Universidade do Estado da Bahia - Programa de Residência Multiprofissional em Oncologia. Farmacêutica do Hospital Santa Izabel - Salvador Bahia. Praça Conselheiro Almeida Couto, 500, Nazaré, Salvador-Bahia, CEP 40050-410. martasfribeiro@ gmail.com.

${ }^{2}$ Mestre em Toxicologia. Professor da Universidade do Estado Bahia - Departamento de Ciências da Vida. Rua Silveira Martins, 2555, Cabula, Salvador-Bahia, CEP 41150-000. farias.mt@gmail.com.

${ }^{3}$ Especialista em Farmácia Hospitalar pela Faculdade Oswaldo Cruz. Especialista em Farmácia Clínica e Hospitalar em Oncologia pelo Instituto Pessoa. Farmacêutica do Hospital Português da Bahia - Salvador Bahia. Rua Alagoinhas, 489, Rio Vermelho, Salvador, Bahia, Brasil. 41940-620. laniaab@gmail.com.

${ }^{4}$ Mestre em Medicina e Saúde. Professora da Escola Bahiana de Medicina e Saúde Pública. Gerente de Ensino e Pesquisa do Hospital Santa Izabel - Salvador Bahia. Praça Conselheiro Almeida Couto, 500, Nazaré, Salvador-Bahia, CEP 40050-410. patricia@ santacasaba.org.br.
} 
ISSN 2179-6750

questionnaire, analyzing the factors that may influence non-adherence, an average of $24.83 \pm 4.84$ points was detected, which corresponds to no difficulty in adherence to treatment. There was a difference in the results, but the valuation methodologies and the elucidation of information extracted differ in such a way that each method must be analyzed separately and criticized within their limitations. It was possible to infer that the Morisky test and Green is useful to guide an assessment, but does not reflect the degree of adherence, since it tends to overestimate the nonadherence. However, review the factors that may influence adherence, as proposed by Marques, is a useful way to detect the difficulties of patients to accede to drug treatment, in order to influence in a positive way the adherence.

Key-words: Breast Neoplasms, Medication Adherence, Evaluation, Cancer Care Facilities, Neoplasms Hormone-Dependent.

\section{Resumen}

La adherencia se define como el grado de cumplimiento de las instrucciones médicas por el paciente. La terapia endocrina o terapia hormonal es el uso de sustancias similares a las hormonas, o suprimir estos, para inhibir el crecimiento del tumor. Es un tratamiento a largo plazo, con efectos adversos significativos, lo que implica dificultades para la adhesión. En este estudio se utilizaron dos métodos para evaluar la adhesión, la prueba de Morisky y Green y el cuestionario propuesto por Marques. De acuerdo con los resultados de la prueba de Morisky y Green, $80 \%$ de los pacientes entrevistados fueron considerados no adherentes, mientras, a través del cuestionario de Marques, en análisis de los factores que pueden influir en la no adhesión, se detectó un promedio de puntos de $24,83 \pm 4,84$, correspondiente al resultado de ninguna dificultad para adherirse al tratamiento. Había una diferencia en los resultados, pero los métodos de evaluación y la elucidación de la información se diferencia hasta el punto que cada método debe ser analizado por separado y criticado dentro de sus limitaciones. Fue posible inferir que la prueba de Morisky y Green es útil para guiar una evaluación, pero no refleja el grado de adhesión, porque tiene tendencia a sobrevalorar la falta de adhesión. Sin embargo, evaluar los factores que pueden influir en la adherencia, de acuerdo con la propuesta de Marques, es una forma útil para detectar las dificultades de los pacientes a adherirse al tratamiento farmacológico y mejorar la adherencia.

Palabras-claves: Neoplasias de la Mama, Cumplimiento de La Medicación, Evaluación, Instituciones Oncológicas, Neoplasias Hormono-Dependientes.

\section{Introdução}

Apesar da evolução no tratamento e do aumento de diagnóstico precoce, o câncer de mama ainda é a principal causa se mortalidade por neoplasia, em mulheres, em todo o mundo ${ }^{1 .}$

As modalidades terapêuticas disponíveis atualmente para o câncer de mama são a cirurgia e a radioterapia para o tratamento loco-regional, e a hormonioterapia e a quimioterapia para o tratamento sistêmico. A hormonioterapia está indicada tanto para as pacientes consideradas com risco mínimo de recorrência da doença como para aquelas com risco elevado. Também há indicação para os casos de tumores não operáveis, em que há impossibilidade de administração de quimioterapia, e como terapia paliativa na doença metastática ${ }^{2}$.

A terapia endócrina ou hormonioterapia consiste no uso de substâncias semelhantes a 
ISSN 2179-6750

hormônios, ou supressoras desses, para inibir o crescimento tumoral. É um tratamento de longa duração e com efeitos adversos importantes, o que implica em dificuldade de adesão e persistência no tratamento ${ }^{3}$.

O tratamento principal envolve o uso de moduladores seletivos que competem pelos receptores órgãos-alvo e/ou drogas que agem como inibitórias por influenciar diretamente a síntese dos hormônios naturais. A esse efeito inibitório sobre a atividade estrogênica normal na mulher, se devem os principais sintomas associados à hormonioterapia no câncer de mama.

Muitos são os fatores que influenciam a não-adesão e conhecê-los é uma importante ferramenta para os profissionais de saúde que acompanham a evolução dos pacientes com câncer ${ }^{6}$. As chances de haver sucesso nos resultados clínicos são bem maiores quando as dificuldades relacionadas ao tratamento são identificadas e tratadas de maneira eficaz ${ }^{4}$.

Este trabalho avaliou a adesão à hormonioterapia oral por usuários com diagnóstico de câncer de mama, em tratamento ambulatorial, por meio de dois instrumentos já validados, com o intuito de levantar estatísticas que possam mediar intervenções futuras em prol do uso adequado de medicamentos em domicílio.

\section{Métodos}

O estudo foi realizado em uma UNACON na cidade de Salvador - Bahia. O trabalho passou por avaliação no Comitê de Ética da própria instituição, sendo aprovado com número CAAE 49157715.0.0000.5520.

A coleta foi realizada na sala de dispensação de medicamentos orais, no ambulatório de oncologia SUS. Para a coleta dos dados foram utilizados três instrumentos: Ficha de Identificação do Paciente; Teste Morisky e Green ${ }^{5}$; e Questionário estruturado por Marques ${ }^{6}$ para avaliar os fatores que podem influenciar a adesão ao tratamento. Todos os pacientes responderam aos três instrumentos simultaneamente.

O teste Morisky e Green, formado por quatro perguntas, com respostas Sim ou Não, é considerado positivo para não-adesão se o paciente responder Sim a pelo menos uma das perguntas.

A escala de Marques avalia através da soma de pontos das respostas dos entrevistados se existe nenhuma, pouca, moderada ou muita dificuldade em aderir ao tratamento medicamentoso. O escorres variam de 17 a 85 pontos, quanto maior o valor, maior a dificuldade de adesão.

O trabalho contou com a participação de 30 pacientes com diagnóstico de câncer de mama, em seguimento ambulatorial, de outubro de 2015 a janeiro de 2016, escolhidos aleatoriamente, conforme disponibilidade para entrevista no ato da dispensação do medicamento. 


\section{Resultados}

Foram entrevistados 30 pacientes em seguimento ambulatorial, com diagnóstico de câncer de mama, sendo 29 do sexo feminino e apenas 1 (3,33\%) do sexo masculino. A idade média dos pacientes entrevistados foi de $55,13 \pm 11,35$ anos. As faixas etárias prevalentes foram dos 36 aos 50 anos de idade e dos 51 aos 75 anos de idade, representando 40\% e 53,3\% dos pacientes entrevistados, respectivamente.

O diagnóstico diferencial apontou o Carcinoma Ductal Invasivo como predominante, conforme representação na Tabela 1. A ressecção cirúrgica foi o principal tratamento, sendo escolha em $100 \%$ dos pacientes. A radioterapia foi opção terapêutica em $80 \%$ dos pacientes e a quimioterapia em 66,6\%, sendo que 93,3\% (28 pacientes) realizaram pelo menos 2 tratamentos sequenciais, excluindo a hormonioterapia. Os principais medicamentos, em uso pelos pacientes entrevistados, foram o tamoxifeno $(70 \%)$ e o anastrozol (30\%). Devido aos estadiamentos clínicos dos pacientes entrevistados (Tabela 2), todos estavam realizando a hormonioterapia do tipo adjuvante, que consiste em um tratamento complementar após a ressecção cirúrgica.

Tabela 1. Diagnóstico diferencial do câncer de mama nos pacientes entrevistados

\begin{tabular}{|ccc|}
\hline Diagnóstico & n & $\%$ \\
\hline Carcinoma Ductal Invasivo & 24 & 80 \\
\hline Carcinoma Ductal In situ & 1 & 3,3 \\
\hline Carcinoma Lobular Invasivo & 1 & 3,3 \\
\hline Não informado & 4 & 13,3 \\
\hline TOTAL & 30 & 100 \\
\hline
\end{tabular}

Fonte: Pesquisa direta

Tabela 2. Estadiamento clínico, pela Classificação $\mathrm{TNM}^{7}$, dos pacientes entrevistados.

\begin{tabular}{|ccccc|}
\hline Estadiamento & Masculino & Feminino & n & \% \\
\hline I & 1 & 9 & 10 & 33,3 \\
\hline II & 0 & 13 & 13 & 43,3 \\
\hline III & 0 & 6 & 6 & 20 \\
\hline Não informado & 0 & 1 & 1 & 3,3 \\
\hline TOTAL & 1 & 29 & 30 & 100 \\
\hline
\end{tabular}

Fonte: Pesquisa direta

Os dois métodos, utilizados para avaliar a adesão dos pacientes à terapia de bloqueio hormonal, o Teste Morisky e Green ${ }^{5}$ e o questionário estruturado por Marques ${ }^{6}$, obtiveram 
ISSN 2179-6750

resultados divergentes como mostram as Tabelas 3 e 4 , respectivamente.

Tabela 3. Resultado do teste Morisky e Gree.

\begin{tabular}{|c|c|c|c|c|}
\hline \multirow{9}{*}{$\begin{array}{l}\text { Você, alguma vez, esquece de tomar seu remédio? } \\
\text { Você, às vezes, é descuidado quanto ao horário de tomar } \\
\text { seu remédio? } \\
\text { Quando você se sente bem, alguma vez, você deixa de } \\
\text { tomar o remédio? } \\
\text { Quando você se sente mal com o remédio, às vezes, deixa } \\
\text { de tomá-lo? }\end{array}$} & \multicolumn{2}{|c|}{ Sim } & \multicolumn{2}{|c|}{ Não } \\
\hline & $\mathbf{n}$ & $\%$ & $\mathbf{n}$ & $\%$ \\
\hline & 21 & 70,0 & 9 & 30,0 \\
\hline & 11 & 36,66 & 19 & 63,33 \\
\hline & & & 30 & 100,0 \\
\hline & & & 30 & 100,0 \\
\hline & \multicolumn{2}{|c|}{ Positivo } & \multicolumn{2}{|c|}{ Negativo } \\
\hline & $\mathrm{n}$ & $\%$ & $\mathrm{n}$ & $\%$ \\
\hline & 24 & 80,0 & 06 & 20,0 \\
\hline
\end{tabular}

Fonte: Pesquisa direta

De acordo aos resultados do teste Morisky e Green ${ }^{5}$, $80 \%$ dos pacientes entrevistados foram considerados não-aderentes, enquanto que, por meio do questionário de Marques ${ }^{6}$, analisando os fatores que podem influenciar a adesão, foi detectada uma média de pontos de $24,83 \pm 4,84$, que corresponde ao resultado de nenhuma dificuldade em aderir ao tratamento.

Os principais fatores associados ao resultado no teste Morisky e Green ${ }^{5}$ (Tabela 3) foram o esquecimento e o descuido com o horário da administração, pois $70 \%$ dos pacientes referiram esquecer-se de tomar o medicamento e 36,66\% responderam ser descuidados quanto aos horários.

Foi possível analisar separadamente os fatores que podem influenciar a adesão, como sugere Marques ${ }^{6}$. A Tabela 04 apresenta alguns fatores associados à quase todos os pacientes, que contribuiu para os resultados aqui apresentados. O fator 8 analisa a dificuldade de comprar os medicamentos, não detectada em $100 \%$ pacientes. Cerca de $97 \%$ dos pacientes entrevistados responderam que concordam totalmente que a equipe de saúde tem ajudado no tratamento com remédios orais, e mais de $93 \%$ dos pacientes relataram comparecer frequentemente às consultas médicas, bem como, têm respeitado o horário da administração do medicamento $(86,66 \%)$. Todos os pacientes $(100 \%)$ discordaram totalmente quanto à dificuldade em tomar o medicamento e concordaram totalmente que realizam a administração mesmo quando se sentem mal.

Esses fatores corroboraram para o resultado observado na média de pontuação obtida do questionário de Marques ${ }^{6}$, caracterizando os pacientes com nenhuma dificuldade em aderir ao tratamento medicamentoso. 
Tabela 4. Fatores que podem influenciar a adesão.

\begin{tabular}{|c|c|c|c|c|c|c|c|c|c|c|}
\hline \multirow{4}{*}{$\begin{array}{l}\text { Os remédios provocam efeitos } \\
\text { adversos indesejáveis }\end{array}$} & \multicolumn{2}{|c|}{$\begin{array}{l}\text { Concordo } \\
\text { totalmente }\end{array}$} & \multicolumn{2}{|c|}{$\begin{array}{l}\text { Concordo em } \\
\text { parte }\end{array}$} & \multicolumn{2}{|c|}{ Indeciso } & \multicolumn{2}{|c|}{$\begin{array}{l}\text { Discordo em } \\
\text { parte }\end{array}$} & \multicolumn{2}{|c|}{$\begin{array}{l}\text { Discordo } \\
\text { totalmente }\end{array}$} \\
\hline & $\mathrm{n}$ & $\%$ & $\mathrm{n}$ & $\%$ & $\mathrm{n}$ & $\%$ & $\mathrm{n}$ & $\%$ & $\mathrm{n}$ & $\%$ \\
\hline & 4 & 13,33 & 12 & 40,0 & 1 & 3,33 & 6 & 20,0 & 7 & 23,33 \\
\hline & 3 & 10,0 & 11 & 36,66 & & & 6 & 20,0 & 10 & 33,33 \\
\hline $\begin{array}{l}\text { Preciso de ajuda para tomar os } \\
\text { remédios }\end{array}$ & & & & & & & & & 30 & 100,0 \\
\hline $\begin{array}{l}\text { Tenho muitos remédios para } \\
\text { tomar }\end{array}$ & 7 & 23,33 & 5 & 16,66 & 1 & 3,33 & 3 & 10,0 & 14 & 46,66 \\
\hline Não sei como tomar os remédios & 1 & 3,33 & & & & & & & 29 & 96,66 \\
\hline O tratamento oral é complicado & & & & & & & 1 & 3,33 & 28 & 93,33 \\
\hline $\begin{array}{l}\text { Tenho dificuldade de lembrar o } \\
\text { dia do re-início do remédio }\end{array}$ & 2 & 6,66 & 1 & 3,33 & & & 3 & 10,0 & 24 & 80,0 \\
\hline $\begin{array}{l}\text { Tenho dificuldade em comprar } \\
\text { os remédios }\end{array}$ & & & & & & & & & 30 & 100,0 \\
\hline $\begin{array}{l}\text { Não sei se devo tomar os } \\
\text { remédios antes, depois ou com } \\
\text { as refeições }\end{array}$ & & & 1 & 3,33 & 2 & 6,66 & 3 & 10,0 & 24 & 80,0 \\
\hline O remédio é difícil de engolir & 1 & 3,33 & 1 & 3,33 & & & & & 28 & 93,33 \\
\hline $\begin{array}{l}\text { Esqueço de comparecer às } \\
\text { consultas }\end{array}$ & & & 2 & 6,66 & & & & & 28 & 93,33 \\
\hline $\begin{array}{l}\text { O tratamento com remédios orais } \\
\text { proporciona menos falta ao } \\
\text { trabalho }\end{array}$ & 24 & 80,0 & 1 & 3,33 & 4 & 13,3 & & & 1 & 3,33 \\
\hline $\begin{array}{l}\text { A equipe de saúde tem ajudado } \\
\text { no tratamento com remédios } \\
\text { orais }\end{array}$ & 29 & 96,66 & 1 & 3,33 & & & & & & \\
\hline $\begin{array}{l}\text { Tenho horários certos para tomar } \\
\text { os remédios }\end{array}$ & 26 & 86,66 & 4 & 13,33 & & & & & & \\
\hline $\begin{array}{l}\text { Confiro o nome e a dose dos } \\
\text { remédios antes de tomar }\end{array}$ & 23 & 76,66 & 3 & 10 & 3 & 10,0 & 1 & 3,33 & & \\
\hline $\begin{array}{l}\text { Guardo os remédios em local } \\
\text { adequado }\end{array}$ & 29 & 96,66 & & & & & 1 & 3,33 & & \\
\hline $\begin{array}{l}\text { Tomo o remédio mesmo quando } \\
\text { me sinto mal }\end{array}$ & 30 & 100,00 & & & & & & & & \\
\hline
\end{tabular}

Fonte: Pesquisa direta

\section{Discussão}

A idade continua a ser um dos mais importantes fatores de risco para o câncer de mama, pois cerca de quatro em cada cinco casos ocorrem após os 50 anos, e mais de $80 \%$ dos cânceres de mama têm origem no epitélio ductal ${ }^{1}$, como demonstrou o presente estudo.

O câncer de mama também acomete o sexo masculino, porém é uma doença rara que representa menos de $1 \%$ de todos os cânceres em homens ${ }^{8}$. Tal informação acompanha o perfil identificado no estudo, em que apenas 1 paciente era do sexo masculino. 
ISSN 2179-6750

Um estudo levantou informações dos Centros de Alta Complexidade em Oncologia (CACONs) cadastrados no Ministério da Saúde no ano de 2003 (n=173). Foram solicitadas informações sobre o número de casos de câncer de mama matriculados anualmente no período compreendido entre 1995 e 2002. O estudo apontou que a maioria dos casos de câncer de mama foram diagnosticados no estádio II $(42,8 \%)^{9}$, assim como os resultados encontrados nesse estudo.

O melhor manejo do paciente com câncer de mama é aquele que é resultado de uma discussão multidisciplinar, envolvendo a participação do cirurgião, do radioterapêutica e do oncologista clínico $^{20,21}$. No carcinoma ductal, a mastectomia é o tratamento curativo para a maioria dos casos, podendo ser conservadora ou não, a depender da extensão da doença ${ }^{21,22}$. Por isso, a cirurgia foi o tratamento curativo de escolha para todos os pacientes desse estudo.

A hormonioterapia adjuvante é empregada após a cirurgia, em pacientes com positividade para expressão de receptores hormonais, tendo grande impacto na sobrevida global ${ }^{20}$. O tamoxifeno continua sendo o tratamento hormonal padrão em mulheres pré-menopausicas. Nas mulheres pósmenopáusicas o tamoxifeno é uma opção adequada, porém, nos últimos anos, os inibidores de aromatase, como o anastrozol, têm surgido como opções de tratamento. Os estudos demonstram consistente redução absoluta de recorrência do câncer de mama nessas mulheres em uso de anastrozol, variando entre $2 \%$ a cerca de $6 \%{ }^{10}$.

Outras opções e combinações terapêuticas na hormonioterapia estão em evidência e em ampla utilização na atualidade. A maior porcentagem do uso do tamoxifeno $(70 \%)$ reflete a realidade das opções terapêuticas disponíveis nos serviços públicos de tratamento oncológico no Brasil.

Existem diversas metodologias para se avaliar a adesão ao tratamento prescrito, porém não há um método considerado padrão-ouro. As entrevistas estruturadas são métodos de ampla utilização, porém com diversas limitações, uma vez que muitas questões subjetivas podem interferir na fidedignidade das respostas dos pacientes ${ }^{11}$. Essa foi uma limitação importante observada nos métodos de avaliação de adesão utilizados nesse estudo.

O teste Morisky e Green ${ }^{5}$ é útil para nortear uma avaliação, porém não reflete bem o grau de adesão dos pacientes, uma vez que tende a superestimar a não-adesão. Isso pode ser observado ao se avaliar o que sugere a primeira pergunta: "Você, alguma vez, esquece de tomar seu remédio?". Um esquecimento esporádico não configura falta de adesão. Alguns fatores associados à mudança de rotina podem fazer com o paciente se esqueça de tomar um comprimido em uma determinada situação. Isso pode ser observado nos $70 \%$ de respostas afirmativas à primeira pergunta.

Estudos mostram que algum grau de não-adesão ocorre universalmente, mesmo em doenças 22 
ISSN 2179-6750

que envolvem potenciais riscos de vida ${ }^{12}$. Isso ficou evidenciado no resultado do teste de Morisky e Green ${ }^{5}$, porém o grau e os fatores influenciadores não são avaliados pelo questionário.

O questionário de Marques ${ }^{6}$ amplia os questionamentos e possibilita analisar separadamente fatores que podem influenciar a adesão à terapia prescrita, porém ainda é limitante e não permite analisar fatores associados às particularidades da doença.

No público em questão não houve dificuldade de acesso aos medicamentos, visto que tais medicamentos são disponibilizados aos pacientes em tratamento, cadastrados nas unidades de atendimento de referência pelo serviço público. A retirada do medicamento é mensal e o paciente ou acompanhante, obrigatoriamente se apresentam ao serviço de farmácia e, desse modo são acompanhados quanto ao uso. No entanto, muitos pacientes não residem na capital e em alguns casos o acesso é dificultado pela distância, podendo assim ocorrer o desabastecimento.

$\mathrm{Na}$ unidade em estudo, os pacientes em hormonioterapia são acompanhados pelo serviço de farmácia. Uma cartilha de orientação é entregue pelo farmacêutico no início do tratamento, em consulta para orientação. As dispensações seguintes são realizadas por estagiários de farmácia, sob orientação profissional.

A elevada não-adesão detectada através do teste Morisky e Green ${ }^{5}$ nesse estudo é questionável diante da sistemática de dispensação no serviço e do resultado obtido pelo questionário de Marques ${ }^{6}$. A orientação e o acompanhamento contínuo de profissionais da saúde, principalmente o farmacêutico, favorecem a adesão ao tratamento, pois na prática da terapia medicamentosa surgem dúvidas que podem ser sanadas durante a retirada mensal dos medicamentos ${ }^{13}$. Estudo semelhante detectou que a falta de comunicação e orientação ao paciente, por parte da equipe de saúde, foi um fator que influenciou os resultados de adesão ${ }^{19}$. Desse modo, os cuidados na dispensação adotados pela instituição, favoreceram o resultado positivo obtido do questionário de Marques ${ }^{6}$.

Os resultados obtidos, a partir desse estudo, não definem qual método é mais confiável ou vantajoso para se avaliar a adesão, identificando as limitações envolvidas nesses tipos de avaliação e levando a um reconhecimento da importância da interpretação e manejo das dificuldades apresentadas pelos pacientes.

Os métodos de avaliação fornecem apenas uma estimativa do comportamento atual de adesão do paciente. No exercício prático, a definição de níveis pontuais de adesão e não-adesão deve ser interpretada com cautela, pois o fenômeno dose-resposta a um tratamento medicamentoso é uma função contínua ${ }^{16}$.

O paciente é referido como um espaço, o lócus onde um medicamento terá sua ação ${ }^{13}$. Nele 23 
ISSN 2179-6750

estão centrados todos os fatores que interferem na adesão à terapêutica, refletindo o contexto individual, familiar e social ${ }^{11}$. Por isso, o acompanhamento individualizado e sistemático é importante para identificar fatores limitantes à adesão terapêutica e nortear o profissional de saúde em suas intervenções.

A adesão a uma terapia envolve uma análise mais complexa, por isso o tempo de relacionamento estabelecido entre o paciente e o profissional de saúde e a habilidade do profissional em formular e interpretar as questões influenciam diretamente o resultado da avaliação ${ }^{15}$.

\section{Conclusão}

Observou-se uma elevada falta de adesão nos pacientes entrevistados, porém a maioria desses não apresentou dificuldade em conduzir seu próprio tratamento. A diferença nos resultados das avaliações, juntamente com as referências de outros estudos, permitiu concluir que cada método deve ser analisado separadamente e criticado dentro de suas limitações.

Foi possível inferir que o teste Morisky e Green ${ }^{5}$ é útil para nortear uma avaliação, porém não reflete bem o grau de adesão, uma vez que tende a superestimá-la. Todavia, avaliar os fatores que podem influenciar a adesão, como proposto por Marques ${ }^{6}$, é uma maneira útil de detectar as dificuldades dos pacientes em aderir ao tratamento medicamentoso e trabalhar sobre essas, a fim de influenciar de maneira positiva a adesão.

A orientação farmacêutica, prévia à utilização inicial da hormonioterapia, contribuiu para a diminuta dificuldade em aderir ao tratamento. Sendo assim, os serviços de saúde que trabalham com a dispensação de medicamentos orais devem se organizar para fornecer uma estrutura, tanto física quanto técnica, que garanta o acesso ao profissional farmacêutico e à orientação segura e documentada.

\section{Referências}

1. Instituto Nacional de Câncer (Brasil). Estimativa 2014: Incidência de Câncer no Brasil. Rio de Janeiro: INCA; 2014.

2. Instituto Nacional de Câncer (Brasil). Controle do câncer de mama. Documento de consenso. MS: Rio de Janeiro; 2004.

3. Brito C, Portelai MC, Vasconcellos MTL. Factors associated to persistence with hormonal therapy in women with breast cancer. Rev Saúde Pública. 2014; 48(2): 284-5.

4. Miaskowski C, Shockney L, Chlebowski RT. Adherence to oral endocrine therapy for breast cancer: a nursing perspective. Clin J Oncol Nurs. 2008; 12(2): 213-21. 
ISSN 2179-6750

5. Morisky DE, Green LW, Levine DM. Concurrent and predictive validity of a self-reported measure of medication adherence. Med Care. 1986; 24(1): 67-74.

6. Marques PAC. Pacientes com câncer em tratamento ambulatorial em um hospital privado: atitudes frente à terapia com antineoplásicos orais e lócus de controle de saúde [dissertação]. São Paulo: Escola de Enfermagem da Universidade de São Paulo; 2006.

7. Instituto Nacional de Câncer (Brasil). TNM: classificação de tumores malignos traduzido por Ana Lúcia Amaral Eisenberg. 6. ed. Rio de Janeiro, 2004.

8. Weiss JR, Moysich KB, Swede H. Epidemiology of male breast cancer. Cancer Epidemiol Biomarkers Prev. 2005; 14 :20-6.

9. Thuler LCS, Mendonça GA. Estadiamento inicial dos casos de câncer de mama e colo do útero em mulheres brasileiras. Ver Bras Ginecol Obstet 2005; 27(11): 656-60.

10. Liedke PER. Hormonioterapia Adjuvante em Câncer de Mama. Rev Bras Oncologia Clínica 2006; 8(3): 23-7.

11. Leite SN, Vasconcellos MPC. Adesão à terapêutica medicamentosa: elementos para a discussão de conceitos e pressupostos adotados na literatura. Ciência \& Saúde Coletiva $2003 ; 8(3): 775-82$.

12. Jordan MS, Lopes JF, Okazaki E, Komatsu CL, Nemes MIB. Aderência ao tratamento antiretroviral em Aids: revisão da literatura médica. In: Teixeira PR, et al. Tá difícil de engolir? São Paulo: Editora Nepaids; 2000.

13. Luz MT. Natural, racional, social: razão médica e racionalidade científica moderna. Rio de Janeiro: Editora Campus; 1988.

14. Oliveira AT, Queiroz APA. Perfil de uso da terapia antineoplásica oral: a importância da orientação farmacêutica. Rev Bras Farm Hosp Serv Saúde 2012; 3(4): 24-9.

15. Mac Laughlin EJ, Raehl CL, Treadway AK, Sterling TL, Zoller DP, Bond CA. Assessing medication adherence in the elderly: which tools to use in clinical practice? Drugs Aging $2005 ; 22(3): 231-55$.

16. Obreli-Neto PR, et al. Métodos de avaliação de adesão à farmacoterapia. Rev. Bras. Farm. 2012; 93(4): 403-10.

17. Brunton LL, Lazo JS, Parkker KL. Goodman \& Gilman: as bases farmacológicas da terapêutica. Porto Alegre: Artmed; 2010.

18. Patrick GL. An introduction to medicinal chemistry. New York: Oxford; 2009.

19. Oliveira RS, Menezes JTL, Gonçalves MGL. Adesão à terapia hormonal adjuvante oral em pacientes com câncer de mama. Revista Brasileira de Cancerologia 2012; 58(4): 593-601. 
ISSN 2179-6750

20. Buzaid AC, Maluf FC, Lima CMR. MOC - Manual de Oncologia Clínica do Brasil. 12. ed. São Paulo: Dendrix; 2014.

21. Barros ACSD, Barbosa EM, Gebrim LH. Diagnóstico e Tratamento do Câncer de Mama. Projeto Diretrizes. Associação Médica Brasileira e Conselho Federal de Medicina; 2001.

22. Gebrim LH, et al. Câncer de mama Tratamento Cirúrgico. Projeto Diretrizes. Associação Médica Brasileira e Agência Nacional de Saúde Suplementar; 2011. 